\title{
IRTS: INFRARED TELESCOPE IN SPACE
}

\author{
TOSHIO MATSUMOTO \\ Department of Astrophysics, Nagoya University, Chikusa-ku, Nagoya, Japan 464-01
}

\begin{abstract}
IRTS is a small cryogenically cooled telescope onboard the small space platform SFU (Space Flyer Unit). SFU will be launched with the new Japanese HII rocket on January 1994 and retrieved by the space shuttle.

The IRTS telescope has an aperture of only $15 \mathrm{~cm}$ diameter, but is optimized to observe diffuse extended infrared sources. Four focal plane instruments are being developed under collaboration between Japan and the U.S.A. IRTS covers a wide wavelength range from near-infrared to submillimeter region, and has a capability for the spectroscopic measurement. Due to newly developed detectors, the sky will be surveyed with very high sensitivities. IRTS will provide valuable data on cosmology, galactic structure, cosmic dust, etc.
\end{abstract}

\section{Introduction}

Infrared observation in space with cold optics is extremely valuable in astrophysics. After the success of IRAS, new missions, such as ISO and SIRTF, are planned. These two are big observatories in orbit and require a huge amount of man power and money. Since the community of infrared astronomers in Japan is so small, we are planning a small cooled telescope onboard the small space platform called the Space Flyer Unit (SFU). Our telescope, IRTS (Infrared Telescope in Space), has only $15 \mathrm{~cm}$ aperture but is specially designed to observe specific objectives, that is, diffuse extended sources.

Small telescopes generally have lower collecting power and spatial resolution than big telescopes. Observation times of the big facilities, however, are shared by many observers and the beam size is fine, resulting in difficulties for the observations which need wide sky coverage. On the other hand, small telescopes are very powerful for this kind of observation if the mission is dedicated and objects are extended compared with the beam size. IRTS is specially designed for this kind of objects and will play a complementary role to the big facilities.

\section{Space Flyer Unit: SFU}

$\mathrm{SFU}$ is a small space platform for experiments in space. Fig. 1 shows IRTS on SFU in space. SFU has an octagonal shape of $4.6 \mathrm{~m}$ diameter and weighs $3,500 \mathrm{~kg}$. Outer sections of octagon are used for many kind of experiments, such as material science, life science, etc. IRTS occupies one section as the only astronomical instrument.

SFU will be launched with the Japanese HII rocket on January 1994 and will be retrieved by the space shuttle. Operation for three months in space is scheduled and 3 weeks are allocated for IRTS observation.

SFU will be put in low inclination (31 degrees) and low earth (500 km) orbit. During IRTS observation SFU is rotated around the axis of the octagon once an

Y. Kondo (ed.), Observatories in Earth Orbit and Beyond, 215-222.

(C) 1990 Kluwer Academic Publishers. Printed in The Netherlands. 


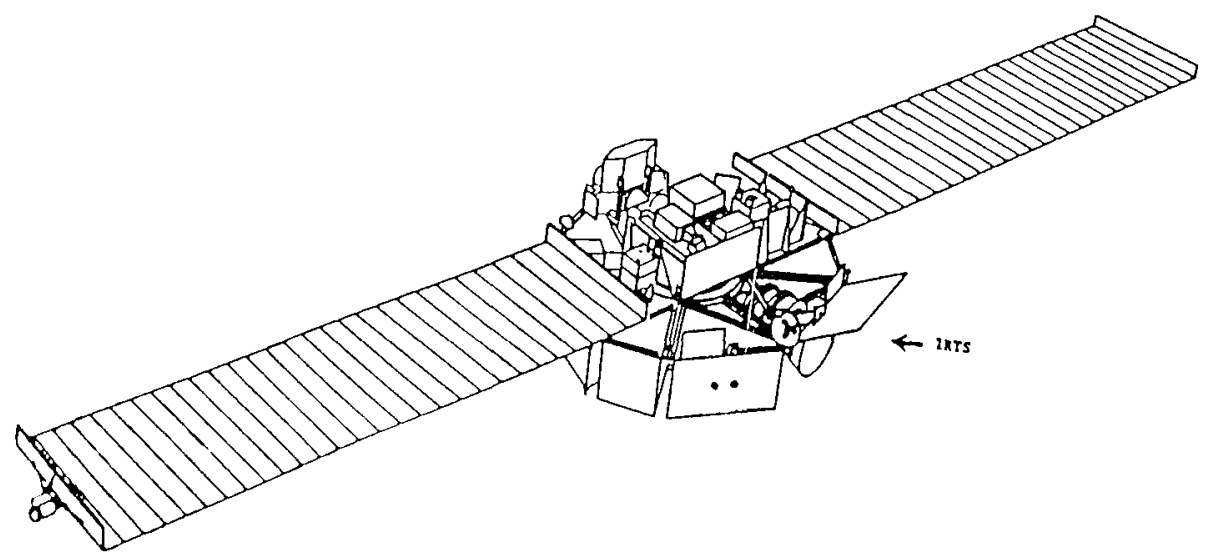

Fig. 1. IRTS on SFU in space.

orbital revolution, so that the direction of IRTS beam is always far away from the sun and the earth's surface. IRTS will survey the sky with constant scan speed and $10 \%$ of the sky is observed during the IRTS mission.

\section{Cryostat and telescope system}

IRTS is a cryogenic system cooled by liquid Helium (Murakami et al. 1989). 100 litres of super fluid Helium is stored in the annular tank which is suspended by the GFRP belts. Phase separation of evaporated gas is made through a porous plug, and the temperature of focal plane instruments is designed to be $1.8 \mathrm{~K}$.

An engeneering model of the IRTS cryostat was fabricated and the required performance of the cryostat was confirmed. The telescope system consists of sunshield, aperture shade, specular cold forebaffle, black cold aftbaffle and telescope. This system is very effective not only to reduce heat load on the cryostat but also to improve baffling performance of the telescope. The telescope itself is $15 \mathrm{~cm}$ Ritchy Cretien system made of aluminum alloy and forms a $\mathrm{F} 4$ beam.

\section{Focal plane instruments}

On the focal plane, four instruments are installed; Near-infrared spectrometer (NIRS), Mid-infrared spectrometer (MIRS), Far-infrared line mapper (FILM), and Farinfrared photometer (FIRP). Development of the focal plane instruments are being carried out under the collaboration of the following institutes; Institute of Space and Astronautical Science, Nagoya University, University of Tokyo, NASA Ames 


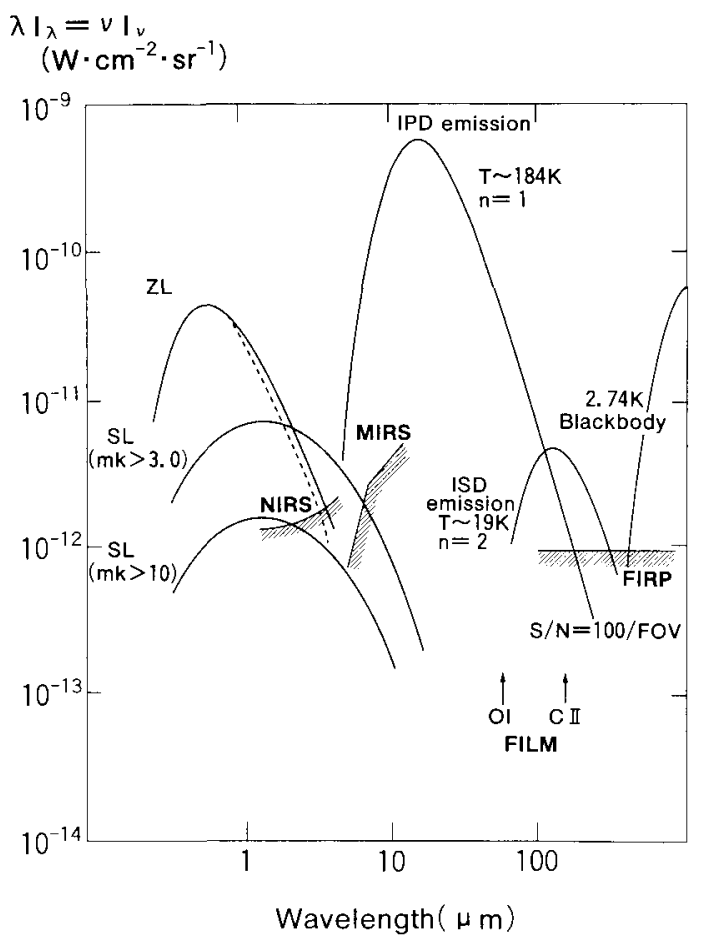

Fig. 2. Infrared surface brightness of the darkest sky and the detection limits of the 4 focal plane instruments.

Research Center, and University of California,Berkeley.

NIRS, MIRS, and FILM use the peripheral area of the beam, while FIRP employs the center of the beam. The star sensor (STS) with an N-slit uses a quarter of the peripheral area of the beam. IRTS is designed to observe diffuse extended objects. Fig. 3 indicates the estimated infrared sky brightness at the darkest region of the sky with the detection limits for IRTS focal plane instruments.

\subsection{NEAR-INFRARED SPECTROMETER: NIRS}

NIRS is a simple grating spectrometer (see Fig. 3) with a 24 element InSb linear array. Characteristic feature of this optical system is a wide field ( 0.12 degree square), wide spectral coverage $(1.2 \mu \mathrm{m}-4.2 \mu \mathrm{m})$, but coarse spectral resolution $(0.12 \mu \mathrm{m}$ band width). A charge integrating amplifier with heated J-FET array is installed (Yamamoto et al. 1989) which results in about 10 times more sensitive detection limit than usual TIA amplifier.

A variety of scientific objectives is expected for NIRS. Interplanetary dust (IPD) scatters sunlight (Zodiacal light: ZL) and emits the thermal radiation in midinfrared region (IPD emission). Spectroscopic observation will delineate the band 


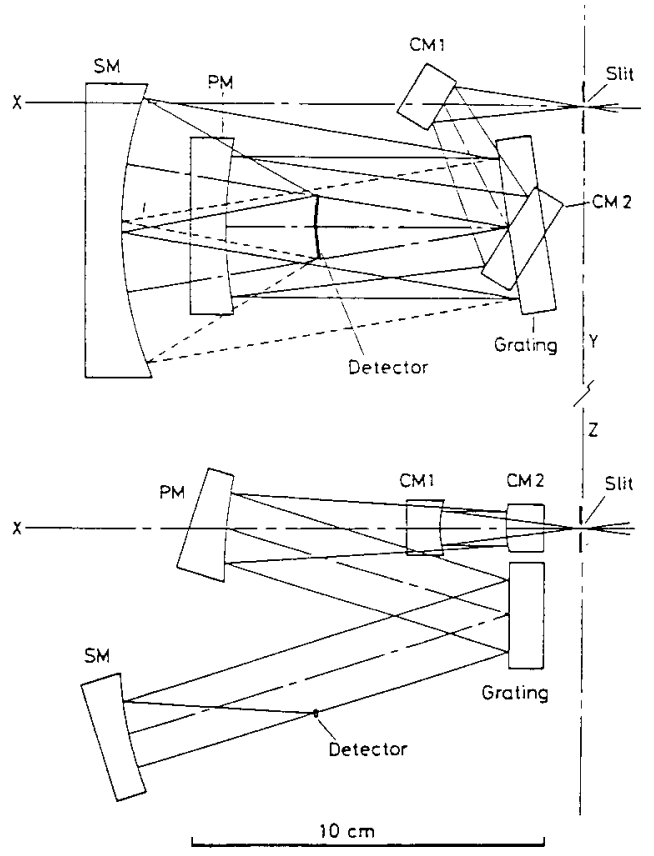

Fig. 3. Optical design of NIRS.

features of the dust, indicating the nature of the dust material.

Star light (SL), that is, an integrated light of the faint stars is also interesting objective. Spectroscopic observation will provide informative data to study the origin of the SL, since important stellar lines of $\mathrm{CO}$ and $\mathrm{H}_{2} \mathrm{O}$ are included in NIRS spectral range. Observation of SL at high galactic latitude is especially important in studying nature of the galactic halo stars. Diffuse extended emission of interstellar matter is also observable. For example, extended emission of the $3.3 \mu \mathrm{m}$ line reported by Giard et al. (1988) will be easily mapped by NIRS.

As is clearly shown in Fig. 2, the sky is very dark at the near-infrared region. This makes the near-infrared region useful as a window to observe extra-galactic diffuse emission. Matsumoto et al. (1988) made a rocket experiment to search for the extragalactic background light (EBL) in the near-infrared region and reported unknown isotropic emission of the order of $10^{-11} \mathrm{~W} \mathrm{~cm}^{-2} \mathrm{sr}^{-1}$ which is possibly attributed to EBL. The observation of the near-infrared EBL is particularly important in the study of the early universe, since the redshifted light of the first generation stars could form EBL in the near-infrared region due to their high redshift. 


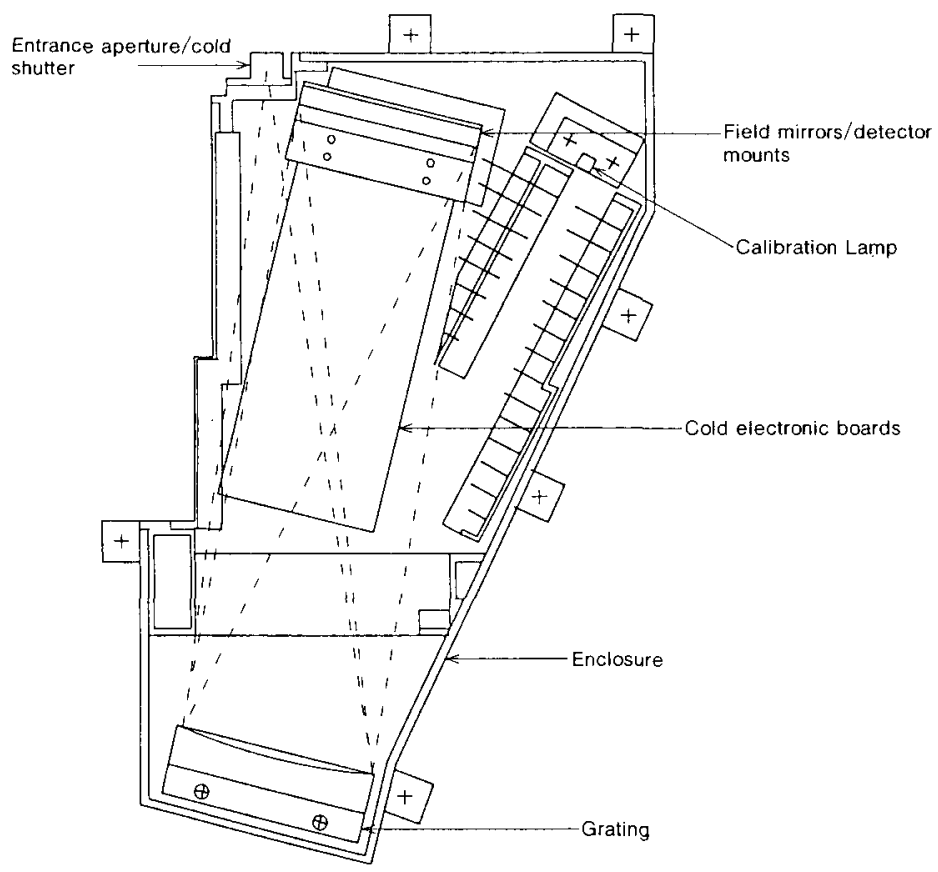

Fig. 4. Optical design of MIRS.

\subsection{Mid-infrared SPECTRometer: MIRS}

MIRS covers wavelength region between $4.3 \mu \mathrm{m}$ and $11.6 \mu \mathrm{m}$ with coarse spectral resolution $(0.3 \mu \mathrm{m}$ bandwidth), and has a beam size of 0.12 degree square. The optical system shown in Fig. 4 is very compact due to the special type of the grating, that is, a variable line spacing, concave, variable blaze angle grating. At the focus a 32 element Si:Ga linear array with feed mirrors is placed. A charge integrating amplifier is used, but signals are multiplexed at the cold plate. The detection limit of MIRS is not due to the read out noise but due to the photon noise of the incident radiation.

NIRS and MIRS are aligned along the scan path with same beam size, which makes it possible to obtain spectral features of the sky over a wide wavelength range. The sky at the MIRS wavelength region is very bright due to IPD emission. Spectral features of the dust emission, such as the $10 \mu \mathrm{m}$ silicate feature, are included in MIRS wavelength range and will be easily identified. Observation of the IPD emission by MIRS will be complementary to the observation of the ZL with NIRS. Combined data will be important in understanding the nature and origin of the IPD.

Dust emission of the interstellar dust (ISD) is also an important target of MIRS. IRAS found the dust emission which has a peak around $10 \mu \mathrm{m}$. This hot dust is 
supposed to be due to fine dust particles (PAH?) heated by a single UV photon and to be closely related with infrared U-lines at $3.3,6.2,7.7,8.6,11.3 \mu \mathrm{m}$. MIRS observations will show how extended hot dust particles are distributed in the interstellar space, and how hot dust is connected with infrared U-lines. MIRS observations will reveal the nature and origin of the hot dust.

Not only extended emission but also point sources will be observed simultaneously. Although aperture of the IRTS telescope is only $15 \mathrm{~cm}$, sensitivity of MIRS for the point source is one order of magnitude higher than LRS of IRAS. Combined data with NIRS will be valuable for studying late type stars and other curious objects.

\subsection{FAR-INFraRed LINE MAPPER: FILM}

FILM has the highest spectral resolution of the IRTS focal plane instruments. FILM is tuned at the fixed wavelength of the $158 \mu \mathrm{m}$ and $63 \mu \mathrm{m}$, corresponding to [CII] and [OI] lines, respectively. Fig. 5 indicates optical design of FILM. A variable spacing, cylindrical concave grating makes the optical design very simple. The entrance slit sets the beam size to be $\left(8^{\prime} \times 20^{\prime}\right)$. Since the ratio of the [CII] and [OI] wavelengths is 5:2, the optical system is set so that [CII] and [OI] lines correspond to 2nd and 5 th order, respectively. Dispersed and focused light is led to the detector system. A beam splitter in the central feed horn reflects the light of the [OI] line and passes the light of the [CII] line. In front of the central feed horn a chopper is installed for modulation. For the [CII] line, three stressed Ge:Ga detectors are employed. One corresponds to the center of the [CII] line with spectral resolution of 430 , and other two detectors are used to observe continuum emission outside of the [CII] line with spectral resolution of 130 . For the [OI] line, a single non-stressed $\mathrm{Ge}: \mathrm{Ga}$ detector is used with spectral resolution of 450 .

Scientific objective of FILM is the physics of the interstellar matter. Recent balloon and airborne observations have revealed that the [CII] line is a key to understanding the photodissociated region. The [OI] line which is originated in more condensed region plays a complementary role to the [CII] line. The recent theory suggests that the [OI] line is important in cooling the slow shock region. Shibai et al. (1990) found diffuse [CII] emission extending to the galactic plane. Since IRTS is extremely powerful in observing extended object, mapping of the galactic plane with the [CII] line will provide valuable data for understanding interstellar physics. Furthermore, due to the high sensitivity of FILM, detection of the [CII] line at high galactic latitude is possible. Since the physical state outside the galactic plane is not well understood, FILM observations will be exciting.

\subsection{Far-Infrared Photometer: FirP}

FIRP is a unique instrument which does not use dispersive optics. The design of FIRP is shown in Fig. 6. The feed horn of FIRP occupies the central part on the focal plane yielding 0.5 degree beam. Incident radiation is divided to 4 channels with beam splitters. 4 composite type bolometers cover the wavelength range between $100 \mu \mathrm{m}$ and $800 \mu \mathrm{m}$ with spectral resolution of $40 \%$. Bolometers of low time 


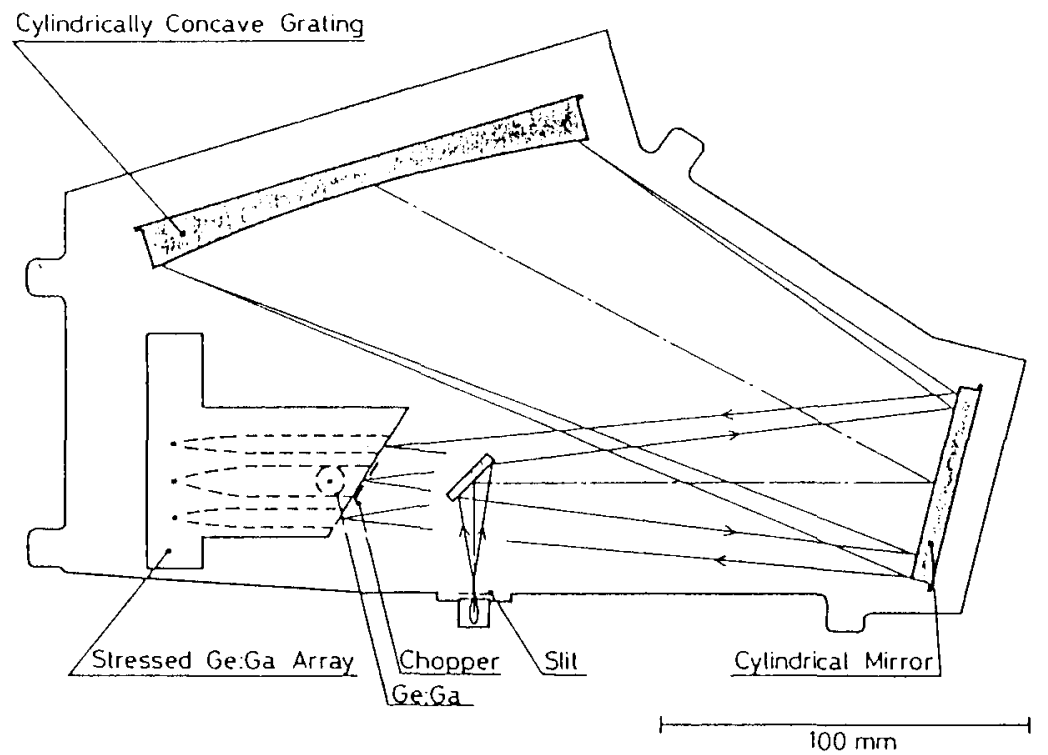

Fig. 5. Optical design of FILM.

constant are used with the $\mathrm{AC}$ biased bridge circuit and are cooled down to $0.3 \mathrm{~K}$ by a Helium 3 refrigerator, resulting in an NEP of $3 \times 10^{-17} \mathrm{~W} \mathrm{~Hz}^{-1 / 2}$.

At the short wavelength side of FIRP, ISD emission is dominant. At high galactic latitude, cirrus emission found by IRAS will be observed with significant accuracy. Compared with FILM data, the physical state of the high latitude clouds will be understood more definitely. In the submillimeter region there exists another "window" which enables us to observe EBL. The sky brightness is very low between ISD emission and the $2.7 \mathrm{~K}$ cosmic background radiation (Lange et al. 1990). Redshifted radiation of the thermal emission from the dust of the first generation forms the EBL in the submillimeter region. This submillimeter EBL is closely related with the near-infrared EBL.

FIRP and NIRS will play complementary role each other and provide valuable information to understand the evolution of the universe after the recombination era. At the longer wavelength side of FIRP, the $2.7 \mathrm{~K}$ cosmic background could be observed. Owing to the high sensitivity, spatial fluctuation, $\Delta T / T$, of the order of $10^{-5}$ for one beam will be detectable. This detection limit will not only provide strong constraints on the fluctuation of the relict radiation, but also makes it able to observe the Sunyaev-Zeldovitch effect for clusters of galaxies and super clusters.

\section{Summary}

IRTS is an explorer type mission which is dedicated and optimized to observe diffuse extended objects. In spite of a small aperture, significant scientific results which can not be attained by the big telescopes are expected. IRTS is the first Japanese infrared telescope in orbit and will be an important mile stone for the infrared astronomy in Japan. 

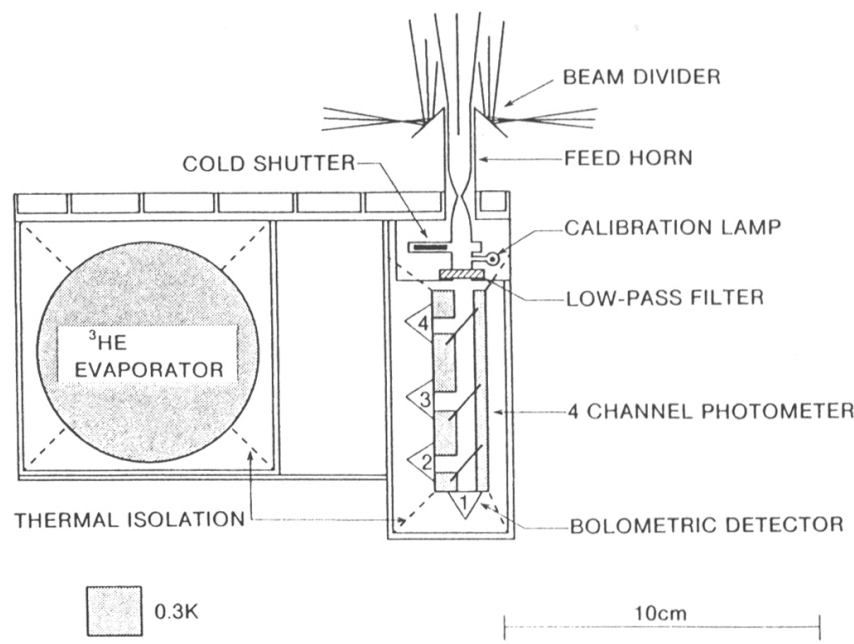

Fig. 6. Schematic view of FIRP.

\section{References}

Giard,M. et al.: 1988, Astron. and Astroph. 201, L1

Lange,A.E. et al.: 1990, Astroph. J., in press

Matsumoto.T., Akiba,M., and Murakami,H.: 1988, Astroph. J. 332, 575

Murakami,M.: 1989, Cryogenics 29, 553

Shibai,H., 1990, submitted to Astroph. J.

Yamamoto,K.: 1989, Proc.SPIE 1157, 338 